\title{
Sequential tunneling current through semiconductor superlattices under intense $\mathrm{THz}$ radiation
}

\author{
Gloria Platero and Ramón Aguado \\ Instituto de Ciencia de Materiales, Cantoblanco, 28049 Madrid, Spain
}

(Received 2 December 1996; accepted for publication 25 April 1997)

\begin{abstract}
Recent transport measurements in GaAs-GaAlAs superlattices under $\mathrm{THz}$ radiation show evidence of dynamical localization and electron pumping in the opposite direction to the dc bias in the linear response regime. The ac field induces absorption and emission processes in the multiwell structure which assist the tunnel and which are reflected as new features in the current density. A calculation within the framework of the Bardeen Hamiltonian has been performed to evaluate the tunneling current through the superlattice in the presence of an ac field. By means of this model these new features are reproduced in the current which can be explained in terms of the new induced tunneling channels and of the charge occupation in the wells. () 1997 American Institute of Physics.
\end{abstract}

[S0003-6951(97)00726-2]

The transport properties of semiconductor superlattices (SLs) have been under intense experimental and theoretical investigation not only under static but also at high frequency fields. ${ }^{1}$ In this work we develop a model to evaluate the sequential current through a GaAs-AlGaAs SL coupled with the radiation by an antenna which produces an electric field intensity of several $\mathrm{kV} / \mathrm{cm}$ and frequencies in the far infrared regime. For finite bias the miniband structure is broken in quasidiscrete well states and the sequential tunneling is the mechanism for the current. It implies memory loss of the electronic wave function which takes place through phonon scattering, interface roughness, or impurities and the characteristic times associated to each of these processes and compared to the tunneling time determine the current density. Therefore there is a finite current between the current peaks corresponding to the alignment of resonant states from consecutive wells because the peaks are smeared due to scattering. In this work we did not include the Coulomb interaction, however we do not expect qualitative changes in the effect of the ac field on the dc current for both the noninteracting and the interacting electron case, at least not for high field intensities. ${ }^{2,3}$ Furthermore, we will analyze the current at low bias where the effect of the charge interaction can be neglected.

In the presence of an ac field the Hamiltonian for a SL consisting of $\mathrm{N}$ wells can be written as:

$$
\begin{aligned}
& H(t)=\sum_{k_{i} \in L, R} E_{k_{i}}(t) \mathbf{c}_{\mathbf{k}_{i}}^{\dagger} c_{\mathbf{k}_{i}}+\sum_{i=1}^{N} \sum_{k_{i}} E_{k_{i}}(t) \mathbf{d}_{\mathbf{k}_{i}}^{\dagger} \mathbf{d}_{\mathbf{k}_{i}} \\
& +\sum_{\substack{i=L \\
k_{i} k_{j} \mid \begin{array}{c}
j=1 \\
i=R
\end{array}}}\left(T_{k_{i} k_{j}} \mathbf{c}_{\mathbf{k}_{i}}^{\dagger} \mathbf{d}_{\mathbf{k}_{j}}+H . c\right) \\
& +\sum_{i=1}^{N-1} \sum_{k_{i} k_{i+1}}\left(T_{k_{i} k_{i+1}} \mathbf{d}_{\mathbf{k}_{i}}^{\dagger} \mathbf{d}_{\mathbf{k}_{i+1}}+H . c\right),
\end{aligned}
$$

where $E_{k i}(t)=E_{k_{i}}^{0}+e F z_{i} \cos \omega_{0} t$ and $z_{i}$ is approximated for

${ }^{\text {a)} E l e c t r o n i c ~ m a i l: ~ r a g u a d o @ e v e r e s t . i c m m . c s i c . e s ~}$ the mean position in the $i$ well, $\omega$ is the field frequency, and $F$ its intensity. The electronic states in each spatial region develop sidebands: $:^{4-6}$
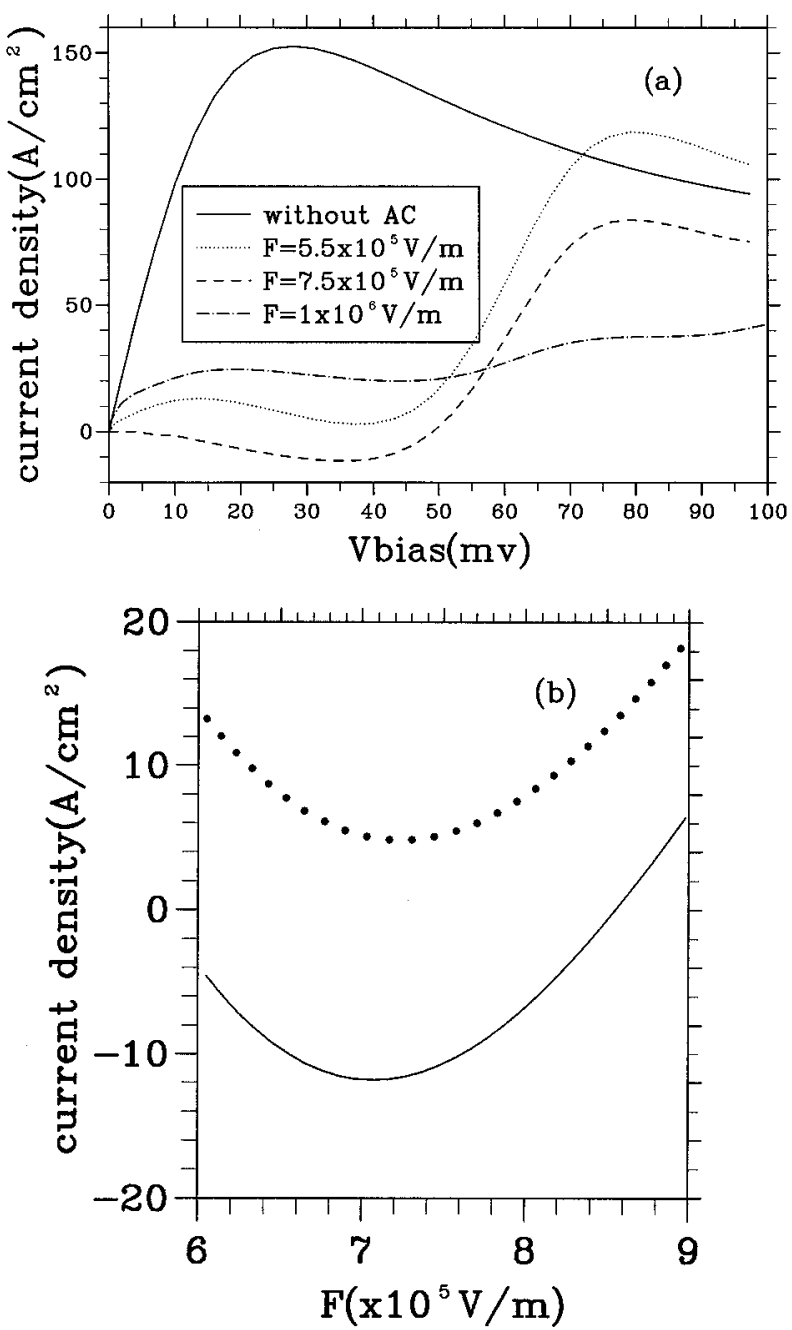

FIG. 1. (a) $J / V$ in the low bias region for different intensities ; $\omega=1.5 \mathrm{THz}$ and $\gamma=1 \mathrm{meV}$. (b) $J / F$ for $V=30 \mathrm{mV}, \gamma=1 \mathrm{meV}$ (continuous line) and $\gamma=2 \mathrm{meV}$ (dotted line). 


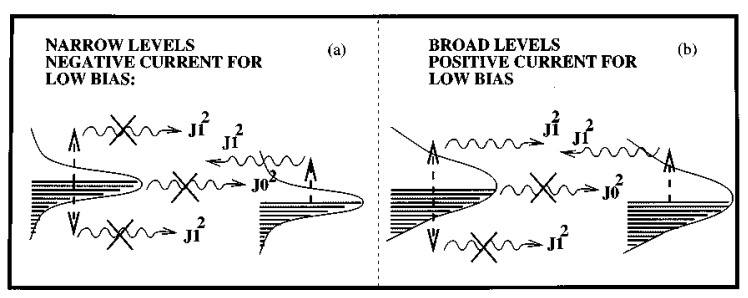

FIG. 2. Schematic drawn of the tunneling mechanism for low bias which produce NC.

$$
\begin{aligned}
\boldsymbol{\Psi}_{\mathbf{k i}}(t) & =e^{-(i / \hbar) E_{k i} t} e^{-\left(i e F z_{i} / \hbar \omega\right) \sin \omega t} \boldsymbol{\Psi}_{\mathbf{k i}} \\
& =e^{-(i / \hbar) E_{k i} t} \sum_{n=-\infty}^{\infty} J_{n}\left(e F z_{i} / \hbar \omega\right) e^{-i n \omega t} \boldsymbol{\Psi}_{\mathbf{k i}} .
\end{aligned}
$$

Following the transfer Hamiltonian ${ }^{7}$ we obtain the transmission probability from one well to the consecutive one, from the emitter to the first well and from the $n$ well to the collector. ${ }^{3}$ From the transmission probability we evaluate the sequential current. The Fermi energies in the wells are then determined imposing current conservation. The density of states (DOSs) in the growth direction is described as Lorentzians of $1-5 \mathrm{meV}$ half width to account for the relaxation between states due to the coupling with phonons $\left(\sim 10^{-13} \mathrm{~s}\right)$. The broadening of the DOS induced by scattering, as well as the two dimensional (2D) continuous in-plane DOS implies that the interwell tunneling is an irreversible process and the Fermi Golden Rule is valid. The expression for the interwell current is:

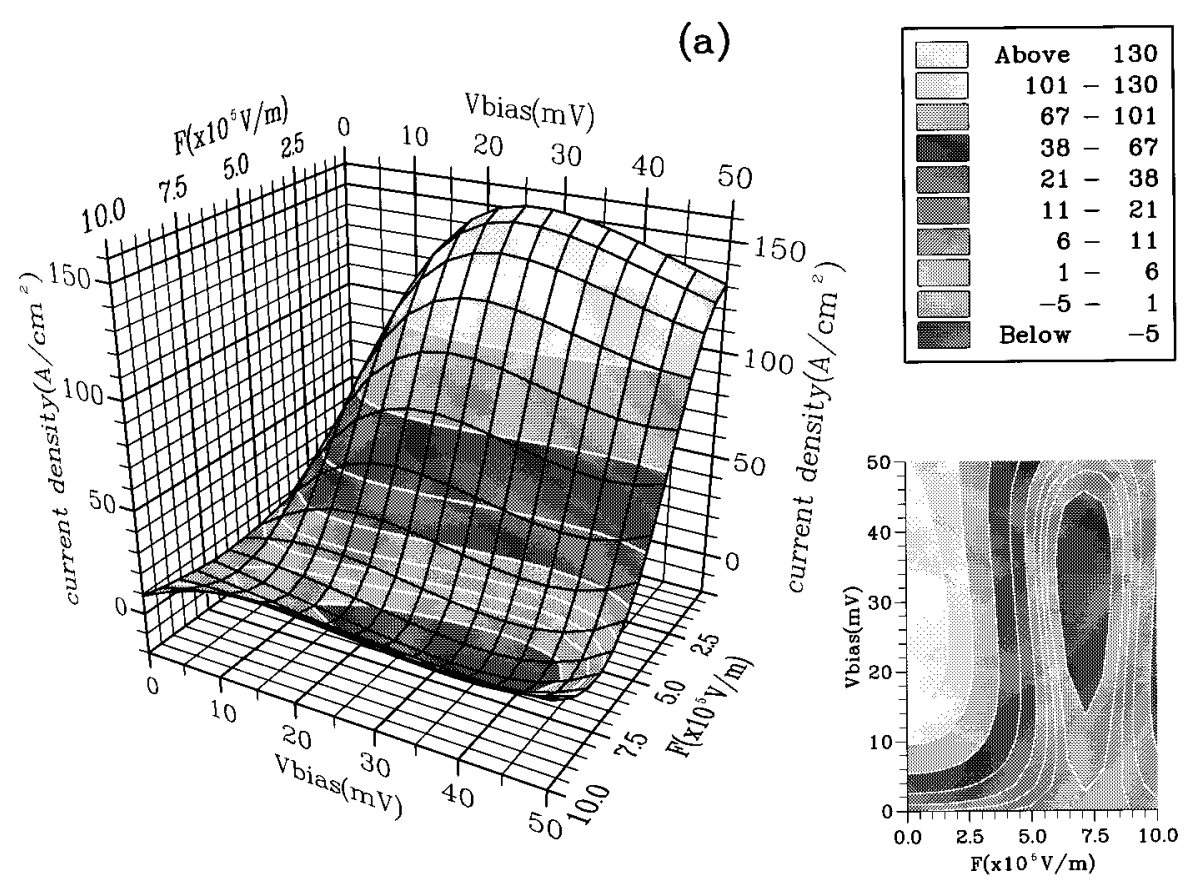

(b)
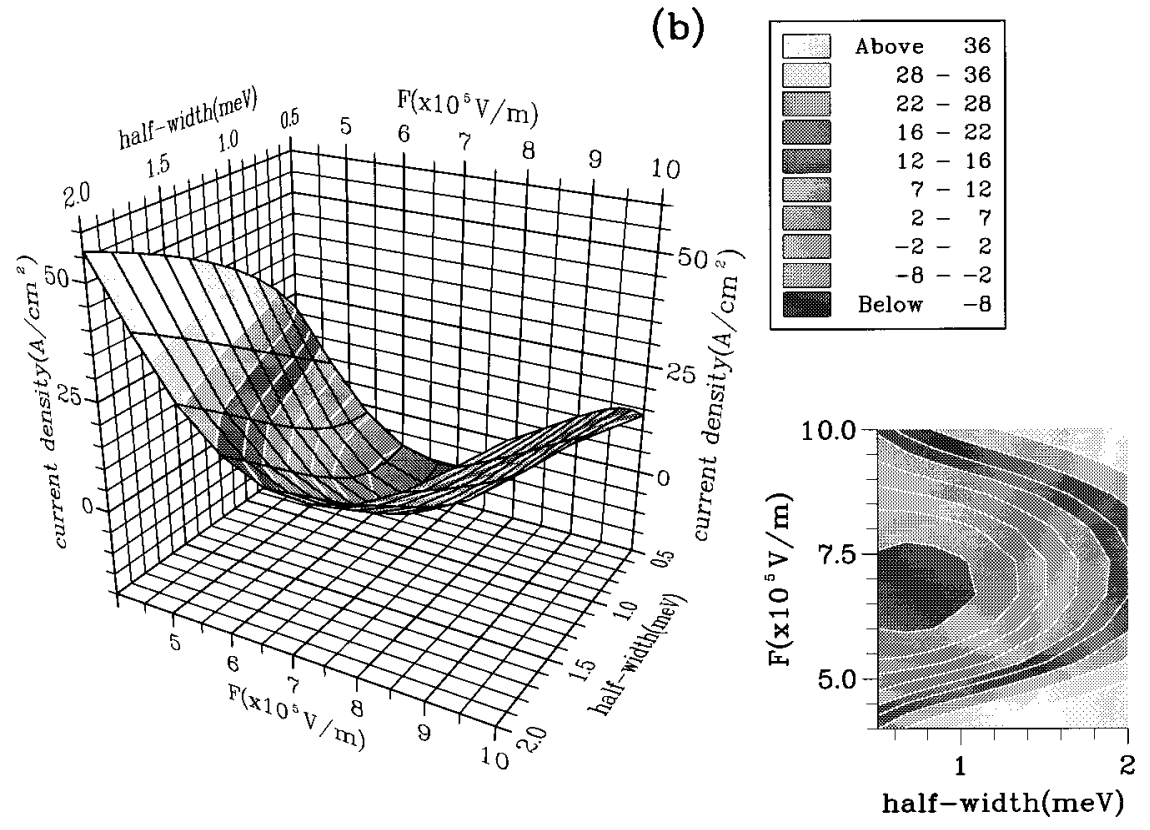

FIG. 3. (a) $J$ vs $F$ and $\gamma$ for $V=30 \mathrm{mV}$, (b) $J$ vs $V$ and $F$ for $\gamma=1 \mathrm{meV}$. 


$$
\begin{aligned}
J_{i, i+1}= & \frac{2 e \hbar k_{B} T}{\pi^{2} m^{*}} \sum_{m=-\infty}^{\infty} J_{m}^{2}\left(\beta_{i, i+1}\right) \\
& \times \int \frac{\gamma}{\left[\left(\epsilon-\epsilon_{r i}\right)^{2}+\gamma^{2}\right]} \frac{\gamma}{\left[\left(\epsilon-\epsilon_{r i+1}+m \hbar \omega\right)^{2}+\gamma^{2}\right]} \\
& \times T_{i+1}(\epsilon, \epsilon+m \hbar \omega) \\
& \times \ln \left(\frac{1+\exp \left(\epsilon_{\omega_{i}}-\epsilon\right) / k_{B} T}{1+\exp \left(\epsilon_{\omega_{i+1}}-\epsilon-m \hbar \omega_{0}\right) / k_{B} T}\right) d \epsilon,
\end{aligned}
$$

where $\epsilon_{r i}$ is the ground state of the $i$ well, $T_{i}(\epsilon, \epsilon+m \hbar \omega)$ is the inelastic transmission through the $i$ barrier, and $\beta_{i, i+1}=e F\left(z_{i+1}-z_{i}\right) / \hbar \omega$ is related to the ac potential drop between the wells. For simplicity we restrict the previous equations to the tunneling between ground to ground state (the generalization to excited well states is straightforward). The current from the emitter to the first well and the one from the $N$ well to the collector are also derived in our model. The Fermi energies $\epsilon_{w i}$ for each well are obtained for each bias voltage through the set of $N+1$ transcendental equations $J-J_{e 1}=0 ; \ldots ; J-J_{i, i+1}=0 ; \ldots ; J-J_{N c}=0$, where $J_{e 1}$ and $J_{N c}$ are the emitter to the left well current and the $N$ well to the collector one respectively, and $J$ is the current to be determined. We have analyzed a SL which consists of 10 undoped wells of GaAs $150 \AA$ wide and 11 barriers of AlGaAs of $50 \AA$ thickness. The emitter and collector are $n$ doped, $n=2 \times 10^{18} \mathrm{~cm}^{-3}$ and the temperature is $T=100 \mathrm{~K}$. We consider an ac field of $\omega=1.5 \mathrm{THz}$ and different $F \sim 10^{5}-10^{6} \mathrm{~V} / \mathrm{m}$. In Fig. 1 (a) $J / V$ is shown in the region of low bias and for different field intensities. We observe (for $F=0$ ) a peak corresponding to the ground to ground state tunnel between the wells. Once the ac field is applied, $J$ is strongly reduced at low bias and even becomes negative. As the bias increases further $\left[F=10^{6} \mathrm{~V} / \mathrm{m}\right.$ in Fig. 1(a)] it becomes positive again. This fact can be explained by means of the wells occupation, the scattering induced broadening of the density of states, and the dynamical localization (DL). The DL consists of the quenching of the direct tunneling channel, i.e., the one weighted by the zero order Bessel function. It happens for $\beta \sim 2.41$, where $\beta$ is the argument of $J_{0}$ for $J_{0}(\beta)=0(\beta=e F d / \hbar \omega$ and $d$ is the SL period $)$. For this structure the DL is reached for $F \sim 7.510^{5} \mathrm{~V} / \mathrm{m}$ and $J$ is inhibited through the central channel. However this channel is open again increasing $F$ as we can see for $F=10^{6} \mathrm{~V} / \mathrm{m}$. In Fig. 1(b) we plot $J / F$ for $\omega=1.5 \mathrm{THz}$ for two different $\gamma$ and a fixed dc bias $V=30 \mathrm{mV}$. For $\gamma=1 \mathrm{meV}$ we observe negative conductance (NC), i.e., the electronic current flows in opposite direction than the applied dc bias. ${ }^{8,9}$ For some ranges of $F$ and $\omega$ the electrons are able to overcome the static bias $\left(e V \leqslant m_{\max } \hbar \omega\right)$ and electronic pumping in the op- posite direction occurs. This effect has been experimentally observed $^{1}$ and can be understood in terms of DL and the charge occupation in the wells. $J$ presents a minima for $F \sim 7.5 \times 10^{5}$ [see Figs. 3(a) and 3(b)] corresponding to the quenching of the direct tunneling channel from ground to ground state. Then the one-photon emission and absorption are the available channels for current but for low bias, the emission is inhibited because the state in the consecutive well is occupied, then absorption is enhanced and $J$ flows towards the emitter. As the $V$ increases the final state is empty and emission is allowed but absorption is inhibited and $J$ flows in the direction of the dc bias. As $\gamma$ increases $[\gamma=2 \mathrm{meV}$ in Fig. 1(b)] the emission channel is opened (see Fig. 2) i.e., there are empty available states in the next well to tunnel and the $J$ flows in the direction of the dc bias. In Fig. 2 we can see schematically the mechanism which produces the negative current. We can see also that as $\gamma$ increases the NC is reduced and eventually destroyed; in Fig. 3(a) we have plotted $J$ as a function of $F$ and $\gamma$ ( $V$ $=30 \mathrm{mV})$ and $J$ as a function of $V$ and $F(\gamma=1 \mathrm{meV})$ for $\omega=1.5 \mathrm{THz}$ [Fig. 3(b)]. The behavior of $J$ for low bias in an ac field agrees very well with the experiments ${ }^{1}$ and we obtain the right position in bias for the features of the current in the presence of the $\mathrm{THz}$ field which in our model verify the Tucker formula, ${ }^{1}$ i.e.,

$$
J_{A C}(V)=\sum_{n=-\infty}^{\infty} J_{n}^{2}(\beta) J_{0}(V+n \hbar \omega / e),
$$

however the position of the measured peak without ac field does not verify (3) and it occurs at higher bias than in our calculations. ${ }^{10}$ This can be due to the fact that we consider some differences in the sample configuration with respect to the experimental one as undoped wells and we consider the noninteracting electrons. Calculations including the Coulomb interaction within a mean field model are in progress.

This work has been supported by the CICYT (Spain) No. MAT 94-0982-c02-02. One of us (R.A) acknowledges financial support from the Comunidad de Madrid.

\footnotetext{
${ }^{1}$ B. J. Keay, S. Zeuner, S. J. Allen, K. D. Maranowski, A. C. Gossard, U. Bhattacharya, and M. J. W. Rodwell, Phys. Rev. Lett. 75, 4102 (1995); S. Zeuner, B. J. Keay, S. J. Allen, K. D. Maranowski, A. C. Gossard, U. Bhattacharya, and M. J. W. Rodwell, Phys. Rev. B 53, 1717 (1996).

${ }^{2}$ J. Fnarrea and G. Platero, Europhys. Lett. 33, 477 (1996).

${ }^{3}$ R. Aguado, G. Platero, M. Moscoso, and L. L. Bonilla, Phys. Rev. B (in press).

${ }^{4}$ P. K. Tien and J. P. Gordon, Phys. Rev. 129, 647 (1963).

${ }^{5}$ M. Wagner, Phys. Rev. A 51, 798 (1995).

${ }^{6}$ J. Inarrea, G. Platero, and C. Tejedor, Phys. Rev. B 50, 4581 (1994).

${ }^{7}$ R. Aguado, J. Inarrea, and G. Platero, Phys. Rev. B 53, 10030 (1996).

${ }^{8}$ G. Platero, R. Aguado, and J. Inarrea, The Physics of Semiconductors (Berlin World Scientific, Berlin, 1996), p. 2263.

${ }^{9}$ Y. Dakhnovskii and H. Metiu, Phys. Rev. B 51, 4193 (1995).

${ }^{10}$ Andreas Wacker, Antti-Pekka Jauho, Stefan Zeuner, and James Allen (unpublished).
} 